\title{
Archivos 2018: Hacia el objetivo de la internacionalización de la revista
}

\author{
Laura Fontcuberta ${ }^{a}$, Jordi Delclós ${ }^{a}$, José Miguel Martinez $z^{a}$, Margarita León ${ }^{a}$, Pilar Varela ${ }^{a}$, Reyes Zapater y Elena Ronda
}

DOI: $10.12961 /$ aprl.2019.22.01.2

Acabamos un año, este pasado 2018, de celebración del 20 aniversario, dedicado, en buena parte ${ }^{1}$, a la publicación de comentarios a una selección de artículos aparecidos en Archivos con el fin de visualizar el avance en el conocimiento y de la práctica profesional durante estas dos últimas décadas; éstos se han dedicado a temas tan relevantes en nuestro campo como las enfermedades profesionales ${ }^{2}$; la vigilancia de la salud ${ }^{3}$; la investigación epidemiológica ${ }^{4,5}$ y la formación en salud laboral ${ }^{6}$ y en medicina del trabajo ${ }^{7}$.

2018 también ha sido un año caracterizado por una mayor internacionalización de la revista. Este compromiso ha estado presente desde los comienzos de Archivos y en la última renovación, en 2016, del Comité Internacional se incorporaron representantes de diferentes instituciones latinoamericanas. La labor de todos ellos en el sentido de dar visibilidad a la aportación de la revista a escala internacional y de aconsejar a los editores, así como realizar el trabajo de evaluación y de redacción de editoriales, ha sido inestimable. Así, contamos con representantes de la Universidad de Puerto Rico; de la Universidad de Costa Rica; de la Universidad Federal de Minas Gerais; de la Superintendencia de Riesgos del Trabajo, de Buenos Aires; y del Instituto Nacional de Salud, de Perú, que se han sumado al resto de miembros. Los nombres de todos ellos pueden consultarse en nuestra página web: http://www.archivosdeprevencion.com, en el apartado de información sobre la revista.

Si hay que destacar también otro hito durante 2018 es el del aumento considerable de evaluadores externos desde el inicio de la revista, puesto que, actualmente, contamos con 333 evaluadores de diferentes orígenes profesionales, especialidades y procedencias geográficas (es importante mencionar que un 15\% aproximadamente son evaluadores extranjeros).

\section{GESTIÓN DE MANUSCRITOS: NÚMERO Y TIPOS DE TRABAJOS}

Empezamos este resumen revisando los manuscritos gestionados durante el último año en relación con los de los dos años anteriores. Se puede visualizar este resumen en la Tabla 1 . Se observa un incremento tanto de los aceptados como del total de los manuscritos gestionados, que hasta ahora se habían mantenido en rangos similares a lo largo de 2016, 2017 y de años anteriores (como ejemplo, la pasada nota editorial, en que además de los años ya citados, se rinde cuentas del año 2015) ${ }^{8}$. En cualquier caso, no podemos sino felicitarnos, y felicitar a nuestros autores, por esta buena noticia.

a. Archivos de Prevención de Riesgos Laborales, Barcelona, España.

Arch Prev Riesgos Labor 2019; 22 (1): 7-10
TABLA 1

Manuscritos $^{\mathrm{a}}$ gestionados en Archivos de Prevención

de Riesgos Laborales (2016 a 2018, volúmenes 19, 20 y 21).

\begin{tabular}{llll} 
& 2016 & 2017 & 2018 \\
\hline Aceptados & 21 & 19 & 28 \\
\hline Rechazados & 13 & 18 & 22 \\
\hline Abandonos & 2 & 4 & 2 \\
\hline En proceso $^{\mathrm{b}}$ & 10 & 1 & 5 \\
\hline Total $^{\text {Tot }}$ & 46 & $\mathbf{5 7}$ & \\
\hline
\end{tabular}

a. Incluye editoriales, originales breves, artículos especiales, revisiones, artículos de opinión, estudios de casos, notas técnicas y cartas al director hasta el 1/12/2018. b. En el momento de elaborarse las Notas Editoriales de los correspondientes años.

En la Tabla 2, se presenta la distribución por tipo de trabajo publicado. Sin embargo, el incremento de trabajos gestionados no ha representado, a su vez, más artículos originales publicados; no obstante, esta modalidad de trabajo sigue teniendo un peso significativo en cada número. El incremento se ha producido, mayoritariamente en los comentarios y artículos especiales.

TABLA 2

Tipos de artículos ${ }^{\mathrm{a}}$ publicados en Archivos de Prevención de Riesgos Laborales (2016 a 2018, volúmenes 19, 20 y 21).

\begin{tabular}{llll} 
& 2016 & 2017 & 2018 \\
\hline Editoriales & 4 & 4 & 5 \\
\hline Notas Editoriales & 1 & 1 & 1 \\
\hline Originales $^{\mathrm{b}}$ & 8 & 6 & 6 \\
\hline Revisiones & 0 & 1 & 2 \\
\hline Estudios de casos & 2 & 1 & 0 \\
\hline Notas Técnicas & 2 & 0 & 0 \\
\hline Cartas a la Directora $^{c}$ & 2 & 5 & 3 \\
\hline Otros $^{c}$ & 2 & 4 & 11
\end{tabular}

a. Excluye secciones informativas (Sociedades, Noticias, Archivos Selección, Ar chivos Evidencia, Archivos Formación).

b. Incluye Originales Breves.

c. Otros: Comunicaciones de la Diada de la SCSL, Artículos Especiales, Artículos de Opinión, etc.

\section{Correspondencia:}

Elena Ronda,

Universidad de Alicante

elena.ronda@ua.es 
TABLA 3

Tiempos de gestión (días) de los manuscritos ${ }^{\mathrm{a}}$ recibidos en Archivos de Prevención de Riesgos Laborales

(2016 a 2018, volúmenes 19, 20 y 21).

2016

2017

2018

\begin{tabular}{lllllll}
\hline & Mediana & Media & Mediana & Media & Mediana & Media \\
\hline Recepción-aceptación & 217 & 153 & 180 & 167 & 102 & 96 \\
\hline Aceptación-publicación & 106 & 81 & 79 & 74 & 52 \\
\hline Recepción-publicación & 323 & 236 & 226 & 242 & 146 & 165 \\
\hline
\end{tabular}

a. Incluye sólo originales, originales breves, revisiones y estudios de casos.

\section{VISITAS A LA REVISTA Y TIEMPOS DE GESTIÓN}

Buenos datos son los referentes a los tiempos de gestión (Tabla 3) que han vuelto a reducirse considerablemente respecto a ediciones anteriores. Estos datos reflejan el esfuerzo de autores, evaluadores externos, secretaria editorial y editores por reducir al máximo los tiempos de gestión. Teniendo en cuenta los datos actuales, no podemos si no felicitarnos por la reducción de esos tiempos de gestión. Otro dato interesante es el de visitas que ha recibido la revista, que ha ascendido a un total de 5.000 desde el 1 de enero hasta el 31 de octubre.

\section{LOS AUTORES Y CONTENIDOS}

De la tabla 4, que nos informa de las características de los autores, nos parece relevante el incremento de autores extranjeros, algo que parece ligar con el aumento de manuscritos gestionados y el proceso de internalización de la revista. Asimismo, si en la edición anterior nos felicitábamos por la incorporación de Castilla y León e Islas Baleares, en esta ocasión, damos la bienvenida a La Rioja, como territorio del autor principal de uno de los manuscritos. Nos gustaría, sin embargo, que estas incorporaciones no supusieran una anécdota, sino que se mantuvieran a lo largo de los números. Positivo es también el dato de unos números más equilibrados entre autores de ambos géneros. Finalmente, y siguiendo con los datos que refleja la Tabla 4, no se observan modificaciones, respecto a otros años, en los centros de investigación y las universidades como instituciones cuyos miembros más aportan al contenido de la revista.
TABLA 4

Características de los autores ${ }^{a}$ (primeros firmantes) que publican en Archivos de Prevención de Riesgos Laborales (2016 a 2018, volúmenes 19,20 y 21).

\begin{tabular}{|c|c|c|c|}
\hline & 2016 & 2017 & 2018 \\
\hline \multicolumn{4}{|l|}{ Género } \\
\hline Hombres & 11 & 6 & 12 \\
\hline Mujeres & 8 & 13 & 14 \\
\hline \multicolumn{4}{|l|}{ Procedencia } \\
\hline Andalucía & 0 & 0 & 1 \\
\hline Aragón & 0 & 0 & 0 \\
\hline Asturias & 0 & 0 & 0 \\
\hline Cantabria & 0 & 0 & 0 \\
\hline Castilla y León & 0 & 1 & 0 \\
\hline Cataluña & 11 & 10 & 13 \\
\hline C. Valenciana & 4 & 3 & 0 \\
\hline Galícia & 0 & 0 & 0 \\
\hline Islas Baleares & 0 & 1 & 0 \\
\hline Islas Canarias & 0 & 0 & 0 \\
\hline Madrid & 2 & 1 & 3 \\
\hline Navarra & 1 & 1 & 1 \\
\hline País Vasco & 1 & 0 & 0 \\
\hline Extremadura & 0 & 0 & 0 \\
\hline Otros países & 0 & 2 & 6 \\
\hline \multicolumn{4}{|l|}{ Institución } \\
\hline $\begin{array}{l}\text { Centros investigación } \\
\text { (incluye universidades) }\end{array}$ & 8 & 5 & 11 \\
\hline Servicios prevención & 2 & 0 & 4 \\
\hline Administración sanitaria & 5 & 4 & 7 \\
\hline Administración laboral & 0 & 2 & 0 \\
\hline Organizaciones sindicales & 0 & 0 & 0 \\
\hline Centros sanitarios & 2 & 5 & 2 \\
\hline Otros & 2 & 3 & 2 \\
\hline
\end{tabular}

a. Excluye secciones fijas (Noticias, Archivos Selección, Archivos Formación). 
En cuanto a los temas tratados (reflejados en la Tabla 5), destacan sobre todo los manuscritos que tratan sobre los problemas de salud de origen laboral, la evaluación de riesgos y la evaluación de intervenciones.

No queremos acabar 2018, sin dejar de mencionar a nuestros evaluadores externos, reconocidos públicamente en el listado que aparece a continuación de esta nota, cuya labor desinteresada y altamente cualificada proporciona el rigor que ha hecho de Archivos una revista de referencia dentro de nuestro ámbito profesional, y a los coordinadores de secciones fijas: Guillermo García, Agueda Giráldez, Amàlia Valls, Silvia Royo, Mar Seguí y Consol Serra. El apoyo de las juntas y socios de la Associació Catalana de Salut Laboral y la Asociación de Medicina del Trabajo de la Comunidad Valenciana es inestimable para la existencia de Archivos. Y como no, sin autores y/o sin lectores, simplemente no podríamos existir. A todos, gracias, y feliz 2019.

TABLA 5

Artículos publicados en Archivos de Prevención de Riesgos Laborales (volúmen 21, año 2018), según contenidos ${ }^{\mathrm{a}}$.

$\begin{array}{lllll}\text { No } 1 & \text { No } 2 & \text { No } 3 & \text { No } 4 & \text { Total } 2018\end{array}$

\begin{tabular}{llllll}
\hline Políticas y estrategias & 1 & 1 & 0 & 1 & 3
\end{tabular}

para la prevención

\begin{tabular}{llllll}
\hline $\begin{array}{l}\text { Evaluación de } \\
\text { intervenciones }\end{array}$ & 0 & 1 & 1 & 2 & 4 \\
\hline
\end{tabular}

\begin{tabular}{llllll}
\hline Vigilancia de la salud & 0 & 1 & 0 & 0 & 1 \\
\hline $\begin{array}{l}\text { Problemas de salud } \\
\text { de origen laboral }\end{array}$ & 2 & 2 & 0 & 1 & 5
\end{tabular}

\begin{tabular}{llllll}
\hline Accidentes de trabajo & 0 & 0 & 0 & 0 & 0 \\
\hline Evaluación de riesgos & 1 & 0 & 3 & 1 & 5 \\
\hline $\begin{array}{l}\text { Investigación y } \\
\text { publicación }\end{array}$ & 0 & 0 & 1 & 0 & 1 \\
\hline $\begin{array}{l}\text { Formación en } \\
\begin{array}{l}\text { Prevención de Riesgos } \\
\text { Laborales }\end{array}\end{array}$ & 0 & 0 & 0 & 2 & 2 \\
\hline
\end{tabular}

a. Excluye secciones fijas (Noticias, Archivos Selección, Archivos Evidencia y Archivos Formación), notas editoriales y comunicaciones de la Diada de la ACSL (antigua SCSL).

\section{BIBLIOGRAFÍA}

1. Benavides FG, García AM, Ronda E. 20 años de investigación en salud laboral... sostiene Archivos (1998-2018). Arch Prev Riesgos Labor 2018; 21: 78-79. doi: 10.12961/aprl.2018.21.02.2.

2. García López V. Para cuándo las Enfermedades Profesionales... decíamos hace 20 años. Arch Prev Riesgos Labor 2018; 21: 85-86. doi: 10.12961/ aprl.2018.21.02.4

3. De Montserrat i Nonó J, Rodríguez-Jareño MC. El mito de Sísifo y la vigilancia de la salud / El mite de Sísif i la vigilància de la salut. Arch Prev Riesgos Labor 2018; 21: 87-89. doi: 10.12961/aprl.2018.21.02.5.

4. Delclòs J. Archivos y la labor invisible de sus directoras y directores: mejorando la capacitación investigadora en salud laboral. Arch Prev Riesgos Labor 2018; 21: 158-159. doi: 10.12961/aprl.2018.21.03.4.

5. Kogevinas M. Estudios de cohorte laboral en España. Arch Prev Riesgos Labor 2018; 21: 160-160. doi: 10.12961/aprl.2018.21.03.5.

6. Ruiz-Frutos C. 20 años de formación en salud laboral: la Administración debería atender la opinión de los expertos. Arch Prev Riesgos Labor 2018; 21: 206-207. DOI: 10.12961/aprl.2018.21.04.5.

7. Serra C. La formación en Medicina del Trabajo. Arch Prev Riesgos Labor 2018; 21: 208-209. doi: 10.12961/aprl.2018.21.04.6.

\section{PERSPECTIVAS DE SUSTITUCIÓN PARA}

EL CR (VI):

¿FUNCIONAN LAS ALTERNATIVAS Y SON

ASEQUIBLES?

14 de enero de 2019, Dortmund (Alemania)

Información:

Svetlana Heller-Hutoran, Bundesanstalt für Arbeitsschutz und Arbeitsmedizin (BAuA)Friedrich-Henkel-Weg 1-25, 44149, Dortmund, Alemania.

Tel.: 0231 9071-2208. Fax: 0231 9071-2611

E-mail: heller-hutoran.svetlana@baua.bund.de Web: https://www.baua.de/DE/Angebote/Veranstaltungen /Termine/2019/01.14-REACH.html

\section{RIESGO ELÉCTRICO:}

¿QUÉ PREVENCIÓN EN LA EMPRESA?

29 de enero de 2019, París (Francia)

Información:

E-mail: electricite2019@inrs.fr

Web: https://www.inrs-electricite2019.fr/

LAS TIC EN LAS SALAS DE CONTROL: USO Y DISEÑO OPTIMIZADOS PARA LA CARGA 7 de febrero de 2019, Dortmund (Alemania)

\section{Información:}

Bettina Lafrenz, Bundesanstalt für Arbeitsschutz und Arbeitsmedizin (BAuA)Friedrich-Henkel-Weg 1-25, 44149, Dortmund, Alemania.

Tel.: 0231 9071-2355

E-mail: lafrenz.bettina@baua.bund.de Web: https://www.baua.de/DE/Angebote/Veranstaltungen /Termine/2019/02.07-Leitwarten.html 


\section{Evaluadores externos para Archivos de Prevención de Riesgos Laborales (2018)}

\section{APELLIDOS, NOMBRE}

Abregú Tueros, Luis Fidel Agún González, Juan José Artime Ríos, Eva María Ballester Laguna, Fernando Barros Dios, Juan Miguel Blanco Bartolomé, Lucía Blanco Donoso, Luis Manuel Brocal Fernández, Francisco Caballero Pérez, Pablo

Calvo-Bonacho, Eva

Campos Fumero, Adriana

Carreras Valls, Rosa

Cortés Pérez, Pere Joan

Esteban Buedo, Valentín

Fernández Luque, Ma. Jesús

Galon, Tanyse

García-Gómez, Montserrat

García González, Guillermo

García Hevia, Ofelia

Garcia Shimizu, Patricia

Gea, Teresa

Giménez Monzo, Daniel

Girao Popolizio, Italo Nicolás

González-Galarzo, M. Carmen

Lezaun Goñi, Mercedes

López López, Juan Carlos

Marhuenda Amorós, Dolores

Moreno-Sueskun, Iñaki

Muñoz Pino, Natalia

Rodríguez Blanes, Gloria Ma.

Ruíz Frutos, Carlos

Sampere Valero, Maite

Santamaría, Carmina

Santaularia Morros, Ana

Santibañez Margüello, Miguel

Seguí Crespo, María del Mar

Sirvent Hernández, Nancy

Tobarra López, Ana

Torá Rocamora, Isabel

Urbaneja Arrúe, Félix

Valmaseda Castellón, Eduard

Vicente Herrero, Ma. Teófila

Villa Sánchez, Fabiola Elena

\section{INSTITUCIÓN}

G.I."Salud en el Trabajo”, Universidad Nacional Agraria de la Selva

Universidad Internacional de Valencia

Hospital Universitario Central de Asturias, Oviedo

Universidad de Alicante

Laboratorio de Radon de Galicia

Universidad de Alicante

Universidad Autónoma de Madrid

Universidad de Alicante

Universidad de Alicante

Ibermutuamur

Instituto Tecnógico de Costa Rica

Institut d'Assistència Sanitària, Salt (Girona)

Institut Català de la Salut, Generalitat de Catalunya

"SGPSP. Direcció General de Salut Pública. Conselleria de Sanitat Universal i Salut Pública"

Comunidad de Madrid. Consejería de Sanidad

Universidade Federal do Triângulo Mineiro (UFTM)

Ministerio de Sanidad, Servicios Sociales e Igualdad

Universidad Internacional de La Rioja

Instituto Asturiano de Prevención de Riesgos Laborales (IAPRL)

"Hospital General Universitario de Alicante de Epidemiología del Servicio de Medicina

Preventiva del Hospital General Universitario de Alicante"

Hospital Sant Joan, Alicante

Universidad de Alicante

Hospital Universitario Araba de Vitoria/Gasteiz

Universidad de Valencia

Instituto de Salud Pública y Laboral de Navarra

MC-Mutual

Universidad Miguel Hernández

Instituto de Salud Pública y Laboral de Navarra

Universidad de Antioquia

"Centro de Salud Pública de Alcoy. Consellería de Sanidad Universal y Salud Pública,

Generalitat Valenciana. Centro de Salud Pública de Alcoy"

Universidad de Huelva

MC-Mutual

Universidad de Montpellier

Departament d'Interior. Generalitat de Catalunya

Universidad de Cantabria

Universidad de Alicante

Universidad de Alicante

Universidad de Alicante

Hospital Clínic de Barcelona

OSALAN

Universitat de Barcelona. Campus de Bellvitge.

Servicio de Prevención Correos. Grupo de Investigación en Medicina del Trabajo (GIMT)

Universidad de Guayaquil

\section{¡Con nuestro reconocimiento y gratitud!}

